\title{
Tecnura
}

\section{Intuición colectiva y estadísticas alternativas para acertar}

\author{
Collective intuition and alternative statistics to hit
Carlos Hildebrando Fonseca Zárate ${ }^{1 \mathbb{D}}$, Juan Carlos García Ubaque ${ }^{2}$ (D), Paula Fernanda García Benítez ${ }^{3}$

Cómo citar: Fonseca Zárate, C.H., García Ubaque, J.C. y García Benítez, P.F. (2020). Intuición colectiva y estadísticas alternativas para acertar Tecnura, 24(63) 104-114. DOI: https://doi.org/10.14483/22487638.16197

Fecha de de recepción: 18 de junio de 2019

Fecha de de aceptación: 18 de octubre de 2019

\section{RESUMEN}

Contexto: El mundo varía rápidamente en el Antropoceno por sistemas socioecológicos sensibles y retroalimentadores. América Latina evidencia distancias grandes entre estadísticas y realidades. Se hacen necesarias mejores herramientas para evaluación y predicción que ofrezcan alternativas esperanzadoras, eficientes y seguras para la sociedad y la naturaleza.

Métodos: Se combinan dos instrumentos de planificación mediante el análisis de componentes principales (ACP): el índice de desarrollo territorial sustentable (IDTS), datos 2000-2010 en Colombia y de sus municipios, departamentos y capital, y el de planificación estratégica participativa con escenarios tridimensionales territoriales (PET2) con respecto a la riqueza intelectual territorial.

Resultados: Se establecieron convergencias y divergencias temáticas entre la realidad estadística (IDTS) y las percepciones, intuiciones e intereses de los agentes, donde las relacionadas con la construcción del conocimiento fueron significativas, en especial frente a los tipos de conocimiento académico/científico, empresarial/ingenieril y ciudadano/ancestral/colectivo.

Discusión: En términos epistemológicos y ontológicos, es necesario conocer la convergencia o divergencia entre la realidad y las estadísticas, y en consecuencia, entre la percepción de los gobernantes y los gobernados. A nivel pragmático, se evidencia que las herramientas planteadas son útiles y de fácil manejo, por lo que su uso (conjunto o separado) puede proveer información valiosa y relevante.

Conclusión: Entender el Antropoceno, en su dinámica de sistema socioecológico, y como sistema social de toma de decisiones, garantiza una mejor comprensión de las necesidades y expectativas de la población, para esto es fundamental revisar los criterios y herramientas de evaluación, seguimiento y toma de decisiones.

\footnotetext{
Ingeniero civil, PhD Geografía. Director, Corporación Simbiosis, Bogotá, Colombia. Contacto: carlosfonsecaz@gmail.com ORCID: https://orcid.org/0000-0003-3653-3899

2 Médico Cirujano, PhD Salud Pública. Profesor titular Universidad Nacional de Colombia, Departamento de Salud Pública. Contacto: jcgarciau@unal.edu.co

ORCID: https://orcid.org/0000-0001-5329-4549

3 Administradora de Empresas Universidad Nacional de Colombia, economista Universidad de los Andes, MSc Salud Pública Universidad de los Andes (C). Contacto: pf.garcia10@uniandes.edu.co.

ORCID: https://orcid.org/0000-0001-7606-851X
} 
INTUICIÓN COLECTIVA Y ESTADÍSTICAS ALTERNATIVAS PARA ACERTAR

Fonseca Zárate, C.H., García Ubaque, J.C. y García Benítez

Palabras clave: desarrollo de la comunidad; indicadores; percepción social; planificación ambiental; planificación participativa.

\section{ABSTRACT}

Context: The world varies quickly in the Anthropocene due to sensitive socio-ecological systems. Latin America evidences great distances between statistics and realities. It is then necessary to have new tools for evaluation and prediction that offer hopeful, efficient and secure alternatives both for society and nature.

Methods: two planification instruments are combined through the Principal Component Analysis (PCA): the "Índice de Desarrollo Territorial Sustentable (IDTS)" with data form 2000-2010 of Colombia and her municipality, department and capital city, and that of "Planificación Estratégica Participativa con Escenarios Tridimensionales Territoriales (PET2)" regarding territorial intellectual wealth

Results: Thematic convergences and divergences were established between the statistical reality
(IDTS) and the perception, intuition and interests of agents, particularly important those related with knowledge construction: academic/scientific, business/engineering and ancestral/collective. Discusion: in a epistemological and ontological level it is necessary to know the convergence or divergence between reality and statistics, and therefor between the rulers and the ruling perceptions. In a pragmatic level it is evident that the established tools are useful and easy to handle so that using them (either together or not) can provide relevant and valuable information.

Conclusion: understanding the Anthropocene, in its dynamic as a socio-ecological and social decision-making system, grants a better comprehension of the needs and expectations of the people. That's why it is necessary to check the criteria and tools for evaluating, follow up and decision-making. Keywords: Comunity Development; Environment Design; Indicators; Participative Planning; Social Perception.

\section{INTRODUCCIÓN}

\section{El complejo, incierto y vertiginoso Antro- poceno}

En la edad de los humanos, Antropoceno, las personas son la fuerza dominante del cambio planetario (Steffen et al., 2015), específicamente en esta época, debido a los desarrollos tecnológicos, la globalización de la producción y del consumo, y el cambio climático, que nos han conducido a ser "un gran mundo en un pequeño planeta" (Elmquist, Cornell, Öhman y Daw, 2014), con transformaciones en la atmosfera, los océanos, los polos, los ríos, las selvas, el clima y la biodiversidad, que eventualmente pueden exceder los límites naturales que determinan la habilidad del planeta físico y biótico de soportar la actividad humana.
En consecuencia, la discusión actual trasciende la dimensión ambiental, propia de décadas anteriores, y se reorienta a replantear el modelo de consumo y apropiación/ acumulación ilimitados, a través de sistemas socioecológicos que, alrededor del uso de los recursos naturales, buscan establecer un límite inferior de condiciones sociales para una vida digna y un límite superior ecosistémico de consumo y desperdicio para la sociedad (Raworth, 2017). De esta manera, cualquier situación por fuera de estos limites es contraria al desarrollo sustentable y, dado que las riquezas del planeta son finitas, si entre los ricos hay mayor velocidad en el incremento en sus ganancias que la del crecimiento, necesariamente los menos ricos están disminuyendo sus ingresos (Piketty, 2014). 


\section{Gestión del conocimiento}

La gestión del conocimiento es el proceso de generación, apropiación, uso, difusión, evaluación y transmisión de saberes; y ha llevado a la consolidación de la economía basada en información (EBI) y de la sociedad del conocimiento. Estas se caracterizan por: globalización de mercados, conocimiento e innovación como ejes de cambios vertiginosos, digitalización y virtualización, convergencia entre información, informática y comunicaciones, conectividad, asimilación del trabajo individual y colectivo a sistemas neuronales, inmediatez y tiempo real para relaciones entre redes, y desintermediación entre productores y consumidores (Tapscott, 1995, citado por Fonseca, Castellanos y Castillo, 2016.).

Sin embargo, el cambio tecnológico ha incrementado la preocupación por el cambio climático, por lo que se requiere una nueva comprensión de sus alcances y mejores instrumentos de lectura de las dinámicas en la cuales se actúa, Entonces, se requiere evolucionar a igual o mayor ritmo que dichos cambios, a partir de herramientas de gestión del conocimiento e innovación social e institucional (Fonseca, Castellanos y Castillo, 2016), centrados en una planificación estratégica y participativa, que reconozca la diversidad dentro de la unidad de propósito y construya gobernanza con eficacia, eficiencia y una correcta relación entre lo público y lo privado.

Con este propósito, se han identificado tres modos de construcción de conocimiento, cuyas características se incluyen en la tabla 1.

Se considera que la sociedad, especialmente en el entorno de las instituciones públicas, se beneficia al permitir la coexistencia balanceada de los tres modos de conocimiento ante el futuro complejo e incierto. En Colom-

Tabla 1. Modos de construcción de conocimiento

\begin{tabular}{cccc}
\hline Modo 1 & Modo 2 & Modo 3 \\
\hline Agentew & Universidad/centro científico & $\begin{array}{c}\text { Parques de ciencia y } \\
\text { tecnología (empresa/ } \\
\text { universidad/Estado) }\end{array}$ & $\begin{array}{c}\text { Estado/investigadores/ } \\
\text { empresas/sociedad civil/ } \\
\text { comunidades/naturaleza }\end{array}$ \\
\hline Método & $\begin{array}{c}\text { Cuantitativo-positivismo/ } \\
\text { racionalismo }\end{array}$ & Cuantitativo/contextual & $\begin{array}{c}\text { Cuali-cuantitativo/Diálogo } \\
\text { intercultural }\end{array}$ \\
\hline Instrumentos & Publicaciones científicas & $\begin{array}{c}\text { Patentes/secreto industrial/ } \\
\text { franquicias }\end{array}$ & $\begin{array}{c}\text { Tradición oral-experiencia/ } \\
\text { talleres }\end{array}$ \\
\hline \multirow{2}{*}{ Aproximación } & Disciplinar especializada & Multi- e interdisciplinar & $\begin{array}{c}\text { Intercultural y } \\
\text { transdisciplinar }\end{array}$ \\
\hline Racionalidad & $\begin{array}{c}\text { Conocer para saber más/ } \\
\text { Enseñanza/aprendizaje }\end{array}$ & $\begin{array}{c}\text { Conocer para vender más/ } \\
\text { competitividad }\end{array}$ & $\begin{array}{c}\text { Conocer para la convivencia } \\
\text { y coexistencia }\end{array}$ \\
\hline \multirow{2}{*}{ Actitud } & Experto docente & Experto facilitador & Construcción colectiva \\
\hline
\end{tabular}

Fuente: Fonseca, Castellanos y Castillo (2016). 
INTUICIÓN COLECTIVA Y ESTADÍSTICAS ALTERNATIVAS PARA ACERTAR

Fonseca Zárate, C.H., García Ubaque, J.C. y García Benítez

bia, son esenciales para el verdadero desarroIlo territorial sustentable, puesto que existen condiciones especiales como el posconflicto y la degradación ambiental, en las que tanto el Estado como la empresa privada y la sociedad civil son fundamentales. Además, se requieren políticas públicas que den respuesta a las necesidades con perspectiva regional (Fonseca Zárate, 2018).

En este sentido, la prospectiva explora el principio según el cual el futuro es múltiple (De Jouvenel, 2000) y depende esencialmente de las decisiones del hombre (Godet y Roubelat, 1996), es decir que, tomando las decisiones correctas en el momento apropiado, se puede construir algo mejor, tanto a nivel personal como social, y es posible incrementar las capacidades individuales y colectivas de prever y modelar el desarrollo preparándose para afrontar y ser resilientes ante diversos escenarios, al diseñar y construir alternativas que permitan un acercamiento progresivo al futuro deseado. En el mundo existen importantes centros de análisis prospectivo que han realizado varios ejercicios en esta dirección, los cuales respaldan su utilidad para la construcción de escenarios posibles y deseables.

\section{MATERIALES Y MÉTODOS}

Mediante la herramienta estadística de análisis de componentes principales (ACP), se realizó un ejercicio de combinación de dos instrumentos de planificación; el primero, de carácter intuitivo colectivo, denominado prospectiva estratégica participativa con escenarios tridimensionales territoriales (PET2), y el segundo, basado en el tratamiento estadístico de información, denominado índice de desarrollo territorial sustentable (IDTS).

EI PET2 corresponde a la fusión, modificada, de la construcción de escenarios futuros posibles, el método Delphi y la construcción interpretativa de gráficos de síntesis. Para los escenarios futuros posibles se incorporó un eje diagonal, orientado a incrementar las perspectivas y la potencia del análisis; para el Delphi, además de las rondas de consultas a pequeños grupos, se incluyó una consulta masiva y para la construcción gráfica se recogieron los dos escenarios más contrapuestos.

El trabajo se llevó a cabo a partir de datos secundarios, y a través de un reprocesamiento de los obtenidos en 2013 por Colciencias. Se adelantó una encuesta masiva sobre necesidades de ciencia, tecnología e innovación en cada departamento y en las quince áreas de conocimiento correspondientes a los programas existentes en Colciencias. El universo estuvo constituido por 23850 personas, de las cuales aproximadamente 11000 eran empresarios y otro tanto investigadores, e incluyó 150 preguntas, las cuales fueron respondidas por casi 6000 personas. Como paso inicial para dicha encuesta, se estableció, mediante talleres con los miembros de los consejos nacionales de programas de ciencia, tecnología e innovación, los diez temas estratégicos que cada uno de ellos consideraba debían acogerse en el ámbito nacional. La consulta masiva se realizó por medios electrónicos con los expertos en cada departamento y en las quince grandes áreas del conocimiento, solicitándoles que escogieran, de la lista nacional de diez temas, los tres que debían trabajarse en su departamento y área de conocimiento, a partir del análisis de dos escenarios: el crítico o turbulento y el más benigno.

Para el ITDS se tomaron los datos recolectados por Fonseca (2018), en los que se establecieron cinco riquezas: humana, intelectual, privada, pública-institucional y natural/ambiental, compuestas por 60 variables, de las cuales 25 son innovadoras, para estimar de manera más integral la riqueza y desarrollo territorial sustentable en los 1122 municipios y 32 departamentos de Colombia. 
La fusión de los dos conjuntos de datos, del IDTS y del PET2, se hizo en el orden departamental, puesto que el ejercicio de consulta nacional permite agregación a ese nivel, teniendo en cuenta que la coincidencia significa que la opinión intuitiva/sujetiva de los encuestados se ajusta a los resultados del subíndice asociado.

Se tomaron en cuenta los datos de los cinco subíndices de riqueza: humano, intelectual, público-institucional, privado y natural am- biental, y se seleccionaron los resultados de los programas de Colciencias más afines a ellos, para explorar la coincidencia o disidencia entre ellos, mediante su agrupación en una sola tabla de datos y la ejecución de un ejercicio de ACP, análisis de componentes principales.

Tabla 2. Prioridades ejercicio PET2

\begin{tabular}{|c|c|c|c|c|}
\hline $\begin{array}{c}\text { Programa/Tema } \\
\text { prioridad }\end{array}$ & Prioridad 1 & Prioridad 2 & Prioridad 3 & Prioridad 4 \\
\hline Sociales y humanas & $\begin{array}{c}\text { Desarrollo humano, } \\
\text { ética y calidad de } \\
\text { vida }\end{array}$ & $\begin{array}{c}\text { Conflicto, } \\
\text { criminalidad, } \\
\text { derechos, justicia, } \\
\text { paz y equidad }\end{array}$ & $\begin{array}{l}\text { Política, estado y } \\
\text { relaciones de poder }\end{array}$ & $\begin{array}{l}\text { Economía, } \\
\text { innovación, } \\
\text { competitividad y } \\
\text { sostenibilidad }\end{array}$ \\
\hline Salud & $\begin{array}{c}\text { Desarrollo de } \\
\text { sistemas y servicios }\end{array}$ & $\begin{array}{c}\text { Promoción, } \\
\text { prevención, calidad } \\
\text { de vida y salud } \\
\text { infantil }\end{array}$ & $\begin{array}{c}\text { Reducción impacto } \\
\text { enfermedades } \\
\text { crónicas }\end{array}$ & $\begin{array}{c}\text { Políticas seguridad } \\
\text { alimentaria y } \\
\text { nutricional, y estilos } \\
\text { de vida saludables }\end{array}$ \\
\hline Educación & $\begin{array}{l}\text { Educación para } \\
\text { la convivencia y } \\
\text { reconciliación }\end{array}$ & $\begin{array}{c}\text { Educación, cultura y } \\
\text { desarrollo }\end{array}$ & $\begin{array}{l}\text { Educación superior } \\
\text { y productividad }\end{array}$ & Educación inicial \\
\hline $\begin{array}{l}\text { Desarrollo } \\
\text { tecnológico e } \\
\text { innovación }\end{array}$ & $\begin{array}{c}\text { Competitividad } \\
\text { sectores estratégicos }\end{array}$ & Producción limpia & $\begin{array}{c}\text { Diseño y } \\
\text { optimización } \\
\text { de procesos } \\
\text { transformación }\end{array}$ & $\begin{array}{l}\text { Innovación en } \\
\text { organizaciones } \\
\text { industria }\end{array}$ \\
\hline Agropecuarias & $\begin{array}{c}\text { Competitividad, } \\
\text { agricultura familiar } \\
\text { y empresarial } \\
\text { mediante CT+i }\end{array}$ & $\begin{array}{c}\text { Articulación } \\
\text { oportunidades } \\
\text { mercado con CT+i }\end{array}$ & $\begin{array}{c}\text { Calidad, cantidad, } \\
\text { variedad e } \\
\text { inocuidad de } \\
\text { los alimentos } \\
\text { disponibles para } \\
\text { la población } \\
\text { colombiana }\end{array}$ & $\begin{array}{c}\text { Conocimiento } \\
\text { apropiado para } \\
\text { condiciones } \\
\text { sociales, } \\
\text { ambientales y } \\
\text { económicas de zona } \\
\text { intertropical }\end{array}$ \\
\hline $\begin{array}{c}\text { Ambiente, } \\
\text { biodiversidad y } \\
\text { hábitat }\end{array}$ & $\begin{array}{c}\text { Gestión integral y } \\
\text { uso sostenible de la } \\
\text { biodiversidad }\end{array}$ & $\begin{array}{c}\text { Gestión integral y } \\
\text { uso sostenible del } \\
\text { agua }\end{array}$ & $\begin{array}{l}\text { Ordenamiento } \\
\text { y planificación } \\
\text { territorial para la } \\
\text { sostenibilidad }\end{array}$ & $\begin{array}{l}\text { Producción más } \\
\text { limpia, consumo } \\
\text { responsable } \\
\text { y tecnologías } \\
\text { ambientales }\end{array}$ \\
\hline
\end{tabular}




\begin{tabular}{|c|c|c|c|c|}
\hline $\begin{array}{c}\text { Programa/Tema } \\
\text { prioridad }\end{array}$ & Prioridad 1 & Prioridad 2 & Prioridad 3 & Prioridad 4 \\
\hline $\begin{array}{c}\text { Electrónica, } \\
\text { telecomunicaciones } \\
\text { e informática }\end{array}$ & $\begin{array}{l}\text { Aplicaciones } \\
\text { TIC en salud y } \\
\text { educación }\end{array}$ & $\begin{array}{l}\text { Aplicaciones TIC } \\
\text { en agroindustria }\end{array}$ & $\begin{array}{l}\text { Competitividad de } \\
\text { empresas con base } \\
\text { tecnológica }\end{array}$ & $\begin{array}{c}\text { Industria inteligente } \\
\text { (IA, control y } \\
\text { automatización) }\end{array}$ \\
\hline Energía y minería & $\begin{array}{c}\text { Fuentes } \\
\text { renovables de } \\
\text { energía eléctrica }\end{array}$ & $\begin{array}{c}\text { Ahorro y uso } \\
\text { eficiente de energía }\end{array}$ & $\begin{array}{l}\text { Desarrollo redes } \\
\text { inteligentes (smart } \\
\text { grids) }\end{array}$ & $\begin{array}{l}\text { Desarrollo de } \\
\text { materiales con valor } \\
\text { agregado a partir de } \\
\text { recursos mineros }\end{array}$ \\
\hline Biotecnología & $\begin{array}{l}\text { Biotecnología } \\
\text { agrícola }\end{array}$ & $\begin{array}{c}\text { Escalado e } \\
\text { ingeniería de } \\
\text { bioprocesos } \\
\end{array}$ & Biorremediación & $\begin{array}{c}\text { Genómica, } \\
\text { proteómica y } \\
\text { metabolómica } \\
\end{array}$ \\
\hline $\begin{array}{c}\text { Mar/recursos } \\
\text { hidrológicos }\end{array}$ & $\begin{array}{c}\text { Biodiversidad } \\
\text { ecosistemas } \\
\text { marinos y costeos }\end{array}$ & $\begin{array}{l}\text { Aprovechamiento } \\
\text { sostenible, recursos } \\
\text { hidrológicos, } \\
\text { marinos, costeros }\end{array}$ & $\begin{array}{l}\text { Calidad ambiental } \\
\text { marina y costera }\end{array}$ & $\begin{array}{c}\text { Amenazas y riesgos } \\
\text { áreas marinas y } \\
\text { costeras }\end{array}$ \\
\hline $\begin{array}{l}\text { Infraestructura, } \\
\text { logística y transporte }\end{array}$ & $\begin{array}{c}\text { Planificación y } \\
\text { diseño óptimo } \\
\text { sistemas } \\
\text { infraestructura, } \\
\text { logística y } \\
\text { transporte }\end{array}$ & $\begin{array}{l}\text { Planificación } \\
\text { regional integral }\end{array}$ & $\begin{array}{l}\text { Nuevos materiales y } \\
\text { formas constructivas } \\
\text { de infraestructura }\end{array}$ & $\begin{array}{c}\text { Diseño, prueba } \\
\text { y adopción de } \\
\text { vehículos y } \\
\text { esquemas operativos } \\
\text { sostenibles }\end{array}$ \\
\hline Vivienda y ciudades & $\begin{array}{c}\text { Diseño y } \\
\text { ordenamiento } \\
\text { urbano y local }\end{array}$ & $\begin{array}{c}\text { Transporte y } \\
\text { movilidad urbana }\end{array}$ & $\begin{array}{l}\text { Gobernabilidad, } \\
\text { seguridad y } \\
\text { convivencia urbana }\end{array}$ & $\begin{array}{c}\text { Economía, } \\
\text { competitividad, } \\
\text { productividad y } \\
\text { empleo urbano- } \\
\text { regional } \\
\end{array}$ \\
\hline Seguridad y defensa & $\begin{array}{c}\text { Seguridad, } \\
\text { defensa, ética y } \\
\text { sociedad }\end{array}$ & $\begin{array}{l}\text { Tecnologías } \\
\text { seguridad } \\
\text { ciudadana, } \\
\text { protección civil. } \\
\text { Bioseguridad, } \\
\text { terrorismo }\end{array}$ & $\begin{array}{c}\text { Materiales } \\
\text { compuestos } \\
\text { en industria de } \\
\text { aviación, civil y } \\
\text { militar }\end{array}$ & $\begin{array}{c}\text { Desarrollo de } \\
\text { sistemas seguridad } \\
\text { telemática y equipos } \\
\text { asociados }\end{array}$ \\
\hline $\begin{array}{c}\text { Deporte, recreación y } \\
\text { actividad física }\end{array}$ & $\begin{array}{c}\text { Ed. física } \\
\text { curricular y } \\
\text { extracurricular, } \\
\text { desarrollo } \\
\text { psicomotor } \\
\text { primera infancia, } \\
\text { adolescencia y } \\
\text { juventud }\end{array}$ & $\begin{array}{l}\text { Pol. pública, } \\
\text { deporte, } \\
\text { recreación, } \\
\text { actividad física, } \\
\text { con enfoque } \\
\text { de derechos y } \\
\text { diversidad }\end{array}$ & $\begin{array}{l}\text { Ciencias básicas } \\
\text { aplicadas a la } \\
\text { actividad física } \\
\text { en relación con } \\
\text { adaptaciones y } \\
\text { efectos sobre la } \\
\text { biología del cuerpo }\end{array}$ & $\begin{array}{c}\text { Evaluación del } \\
\text { impacto de la } \\
\text { promoción del } \\
\text { deporte, recreación, } \\
\text { actividad física y } \\
\text { aprovechamiento } \\
\text { del tiempo libre en } \\
\text { la salud y estilos } \\
\text { vida }\end{array}$ \\
\hline
\end{tabular}

Fuente: Fonseca, Castellanos y Castillo (2016). 


\section{Variables factor map (PCA)}

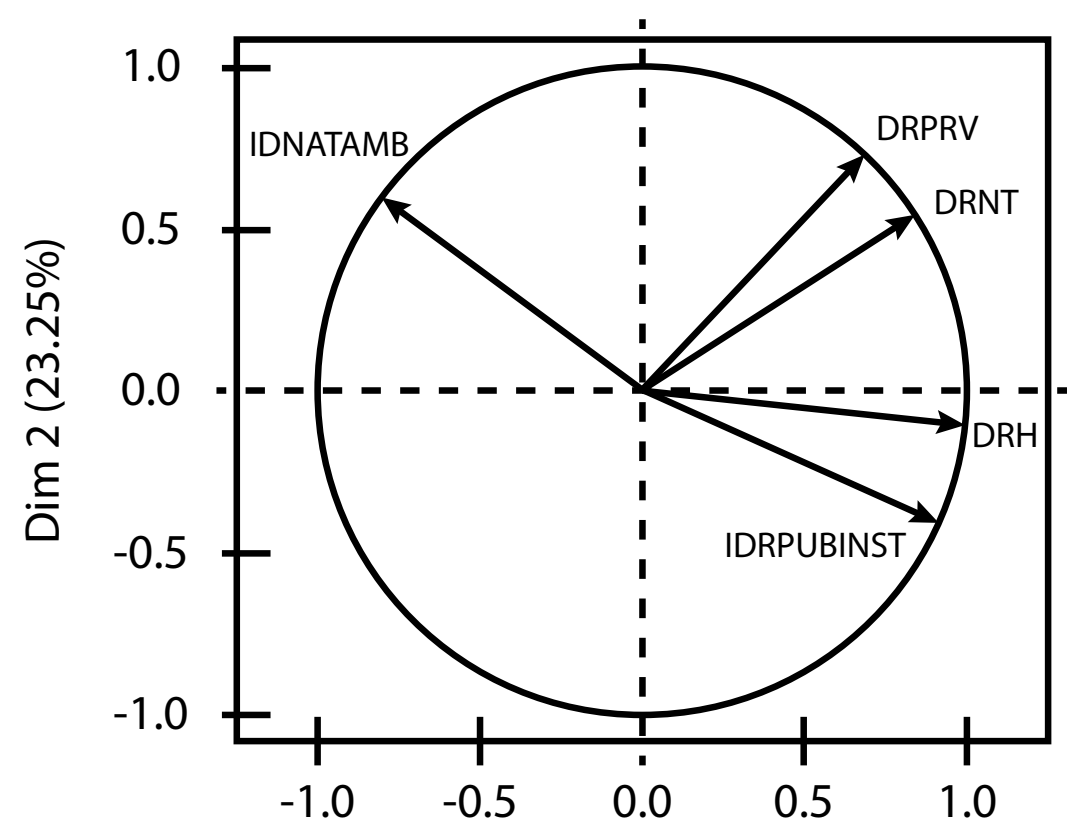

$\operatorname{Dim} 1$ (64.34\%)

Figura 1. Suma de las cinco riquezas territoriales en el ámbito departamental

Fuente: Fonseca Zárate (2018).

La figura 1, representa la suma de las riquezas departamentales establecidas en el ejercicio del ITDS. Como se aprecia, la flecha que corresponde al índice de riqueza natural y ambiental (IDRNATAMB) no se encuentra alineada con las otras cuatro riquezas, lo que indicaría que el progreso o crecimiento en Colombia puede estar sacrificando los recursos naturales y el ambiente.

Esto es importante, en especial en relación con el índice departamental de riqueza intelectual (IDRINT), que agrupa tres modos de construcción del conocimiento: científico/académico, empresarial y ciudadano/ ancestral; el cual al parecer se opone parcialmente (más de 90 grados) al de riqueza natural y ambiental, lo cual significaría que el conocimiento no se está aplicando adecuadamente a los recursos naturales y calidad ambiental, sino que los podría estar afectando negativamente.

También, Ilama la atención el hecho de que el índice departamental de riqueza pública e institucional (IDRPUBINST), se encuentra en dirección casi que totalmente contraria al IDRNATAMB, lo cual significaría que el Estado y las instituciones departamentales estarían afectando negativamente la riqueza natural y ambiental.

Esto puede ser muy controversial, por cuanto existen diversas autoridades y organizaciones ambientales en cada departamento, cuyo propósito es proteger los recursos naturales y la calidad ambiental.

En relación con la combinación de los dos resultados, la tabla 3. presenta la síntesis correspondiente. 
Tabla 3. Resultados del ejercicio de combinación IDTS-PET2, departamentos de Colombia

\section{Ejercicio combinación IDTS-Resultados encuesta PET}

\begin{tabular}{|c|c|c|c|c|c|}
\hline $\begin{array}{l}\text { Programa/ } \\
\text { Riqueza }\end{array}$ & Humana & Intelectual & $\begin{array}{c}\text { Pública/ } \\
\text { institucional }\end{array}$ & Privada & $\begin{array}{c}\text { Natural/ } \\
\text { ambiental }\end{array}$ \\
\hline Ciencias básicas & Coincidencia & $\begin{array}{c}\text { Disidencia } \\
\text { (modo 3) }\end{array}$ & & & $\begin{array}{l}\text { Disidencia } \\
\text { (riesgos) }\end{array}$ \\
\hline $\begin{array}{l}\text { Sociales/ } \\
\text { humanas }\end{array}$ & Coincidencia & $\begin{array}{c}\text { Disidencia } \\
\text { (modo 3) }\end{array}$ & & & \\
\hline Educación & Coincidencia & $\begin{array}{c}\text { Disidencia } \\
\text { (modos } 1 \text { y } 3 \text { ) }\end{array}$ & & & Coincidencia \\
\hline Salud & Coincidencia & & Coincidencia & & \\
\hline Deportes & Coincidencia & & & & \\
\hline Agropecuaria & & $\begin{array}{l}\text { Disidencia } \\
\text { (modo 3) }\end{array}$ & & $\begin{array}{l}\text { Disidencia } \\
\text { (producción) }\end{array}$ & \\
\hline Ambiente & & $\begin{array}{l}\text { Disidencia } \\
\text { (modo 3) }\end{array}$ & Coincidencia & & Coincidencia \\
\hline Infraestructura & & & Coincidencia & & \\
\hline Biotecnología & & & & & Coincidencia \\
\hline $\begin{array}{c}\text { Mar/ } \\
\text { hidrobiología }\end{array}$ & & & & & $\begin{array}{l}\text { Disidencia } \\
\text { (riesgos) }\end{array}$ \\
\hline Vivienda/urbano & & & & $\begin{array}{l}\text { Disidencia } \\
\text { (producción) }\end{array}$ & \\
\hline Electrónica & & & & $\begin{array}{c}\text { Disidencia } \\
\text { (producción) }\end{array}$ & \\
\hline Energía/minería & & & & $\begin{array}{c}\text { Disidencia } \\
\text { (producción) }\end{array}$ & \\
\hline $\begin{array}{l}\text { Des. tecno/ } \\
\text { industria }\end{array}$ & & & & $\begin{array}{l}\text { Disidencia } \\
\text { (producción) }\end{array}$ & \\
\hline
\end{tabular}

Fuente: Elaboración propia. 
INTUICIÓN COLECTIVA Y ESTADÍSTICAS ALTERNATIVAS PARA ACERTAR

Fonseca Zárate, C.H., García Ubaque, J.C. y García Benítez

\section{DISCUSIÓN}

Como se aprecia, el ejercicio arrojó resultados interesantes:

- La riqueza humana del IDTS presenta coincidencia con la opinión de los encuestados en ciencias básicas, sociales, salud, educación y deportes y recreación. Es decir que los temas identificados en los ejercicios Delphi en estos campos apuntan a fortalecer las variables de riqueza humana en estos territorios.

- La riqueza intelectual presenta, en cambio, disidencia con ciencias básicas, sociales, agropecuarias y ambiente, especialmente respecto al modo 3 de conocimiento, que es el relacionado con el diálogo de saberes o conocimiento abierto, en el que la sabiduría ancestral, la complejidad del ordenamiento territorial y la diversidad cultural pueden estar desempeñando un rol significativo. En consecuencia, tal vez sea necesario trabajar más en los mecanismos de interacción de las diversas formas de construcción del conocimiento y, sobre todo, concertar con los diferentes grupos de interés involucrados, en particular con las etnias y grupos culturales.

- La riqueza intelectual también presenta conflicto, disidencia, con ciencias de la educación en cuanto al modo 1 de conocimiento, que es el académico/científico, por cuanto la investigación en pedagogía y enseñanza no se correspondería con los resultados esperados. Algo similar ocurre con el modo 3, donde es válido incrementar los esfuerzos por garantizar más aproximación intercultural en el proceso educativo a nivel departamental; a la vez que valdría la pena preguntarse si, tal vez de manera errónea, la educación actual se está dirigiendo hacia el éxito personal y empresarial como objetivo central, pasando por alto elementos que han sido calificados como centrales, entre ellos: la ciudadanía o la realización personal.

- Es significativo que en ambiente y agropecuario haya disidencia del modo 3 de conocimiento (ancestral/ ciudadano) en el sentido que es parte de la percepción global el que los indígenas han convivido en la selva tropical húmeda y son quienes la han conservado gracias a las prácticas culturales de explotación de carácter agroecológico.

- La riqueza pública e institucional presenta coincidencia con ciencias sociales, salud, infraestructura y ambiente, lo cual podría mostrar que a medida que se consolidan los departamentos en sus aspectos tangibles e intangibles, aumenta el interés y la investigación en esos campos. Sin embargo, una revisión más detallada, que tome en cuenta variables individuales de dicha riqueza, como el índice de desconcentración de la propiedad privada como un factor tangible de la riqueza pública e institucional, podría llegar a revelar otras realidades, por lo que sería razonable incluir estos aspectos en ejercicios posteriores de investigación.

- La paradoja que se evidencia del eventual enfrentamiento de la riqueza pública e institucional con la riqueza natural y ambiental en el IDTS es preocupante, pues revela que el Estado podría estar alineado con un enfoque netamente extractivo, sacrificando necesidades futuras de supervivencia.

- La riqueza natural y ambiental presenta coincidencia con educación, biotecnología, pero disidencia con ciencias del mar y con ciencias básicas, especialmente en 
cuanto a los factores de riesgo. Tanto en el entorno marino como en el terrestre, es necesario profundizar más el conocimiento sobre los peligros y las estrategias para afrontarlos, en especial desde el conocimiento, la tecnología y la innovación social e institucional. En el caso de las ciencias básicas, un bajo conocimiento de la biodiversidad puede dificultar la protección y promoción de la vida en todas sus formas.

- La riqueza privada presenta disidencia con los programas de vivienda, electrónica, energía y minería, desarrollo tecnológico e innovación, y agropecuario en el componente de capacidad productiva orientada a los mercados nacionales. Dado que se crearon nuevos indicadores de la riqueza privada, como el flujo intradepartamental (que interpreta el flujo entre los municipios y la capital departamental), la rentabilidad catastral (que es la división entre el PIB municipal y el valor catastral de todos los predios registrados en el municipio), así como la diversidad productiva y la densidad empresarial, es posible que este resultado refleje la falta de inversión y conocimiento en la transformación empresarial para aumentar la productividad y competitividad, cuestión que merece ser más estudiada.

\section{CONCLUSIONES}

En síntesis, este ejercicio permite revelar posibles conflictos y desencuentros, lo mismo que ausencias temáticas en los programas de $\mathrm{CT}+\mathrm{i}$ sobre aspectos que registra el IDTS y que son relevantes para el desarrollo del país y sus departamentos, y se relacionan con la pertinencia social del conocimiento (García-Ubaque, 2015).
Aunque el futuro es complejo e incierto, puede también ser emocionante; las innovaciones tecnológicas pueden conducir a innovaciones sociales e institucionales favorables. Así, es posible construir sociedades más humanas, incluyentes, equitativas, sustentables y ecoproductivas.

Los métodos de escenarios futuros posibles y de consultas de expertos tipo Delphi son instrumentos pertinentes, de carácter participativo y estratégico, para la toma de decisiones. El método de consultas amplias de expertos, enriquecido con el triaxial de escenarios futuros posibles, permite una visión más amplia del futuro, sin castigos de eficiencia y rapidez. Por esta razón, estos métodos, aplicados de forma periódica, pueden establecer una retroalimentación adecuada para garantizar la gestión del conocimiento, diferenciada regional y sectorialmente, pues es clara la necesidad de usar sus resultados para la toma de decisiones organizacionales, especialmente en el sector público cuya falta de legitimidad social, particularmente en áreas como la salud y el ambiente, resultan preocupantes y dan una sensación de crisis permanente (Agudelo et al., 2016; Morales-Sánchez y García-Ubaque, 2017).

\section{REFERENCIAS}

Agudelo-Calderón, C.A., García-Ubaque, J.C., Robledo-Martínez, R., García-Ubaque, C.A. y Vaca-Bohórquez, M.L. (2016). Identification of capacities in environmental health from environmental authorities in Colombia. Revista de Salud Pública, 18(4), 605-616.

De Jouvenel, H. (2000). A brief methodological guide to scenario building. Techno- 
logical Forecasting and Social Change, 65(1), 37-48.

Elmquist, T., Cornell, S., Öhman, M.C. y Daw, T. (2014). Global Sustainability \& Human Prosperity. Copenhague: Norden.

Fonseca, C. (2018). Lineamientos y sugerencias para el diseño y evaluación de políticas y estrategias de ciencia, tecnología e innovación regionalmente diferenciadas para el desarrollo territorial sustentable de Colombia. Bogotá, D.C.: UPTC-IGAC.

Fonseca Zárate, C.H. (2018). Lineamientos y métodos de construcción y evaluación de políticas y estrategias de ciencia, tecnología e innovación diferenciadas regionalmente para el desarrollo territorial sustentable de Colombia. [Tesis doctoral]. Universidad Pedagógica y Tecnológica de Colombia, Tunja.

Fonseca, C., Castellanos, F. y Castillo, L. (2016). Nuevos métodos de gestión del conocimiento: consulta nacional de administradores públicos con prospectiva participativa. Administración y Desarrollo, 46(2), 175-199

García Ubaque, C.A. (2015). Editorial. Tecnura, 19(46), 11-12.

Godet, M. y Roubelat, F. (1996). Creating the future: the use and misuse of scenarios. Long Range Planning, 29(2), 164-171.

Morales-Sánchez, L.G. y García-Ubaque, J.C. (2017). Gestión de la crisis del sistema de salud en Bogotá. Revista de Salud Pública, 19, 129-133.
Piketty, T. (2014). El capital en el siglo XXI. Bogotá: Fondo de Cultura Económica

Raworth, K. (2017). Doughnut economics: Seven ways to think like a $21 \mathrm{st}$ century economist. Vermont: White River Junction.

Steffen, W., Richardson, K., Rockstrom, J., Cornell, S., Fetzer, I., Benett, E., ..., Sorlin, S. (2015). Planetary boundaries: Guiding human development on a changing planet. Science, 347(6223), 125

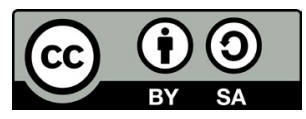

